\title{
Novel contrast mixture achieves contrast resolution of human bladder wall suitable for T1 mapping: applications in interstitial cystitis and beyond
}

\author{
Pradeep Tyagi ${ }^{1}$, Joseph Janicki ${ }^{2}$, Chan-Hong Moon ${ }^{3}$, Jonathan Kaufman ${ }^{2}$, and Christopher \\ Chermansky ${ }^{1}$ \\ 1Department of Urology, University of Pittsburgh, E313 Montefiore Hospital, 3459 Fifth Ave, \\ Pittsburgh, PA 15213, USA \\ ${ }^{2}$ Lipella Pharmaceuticals Inc, Pittsburgh, PA, USA \\ ${ }^{3}$ Department of Radiology, Magnetic Resonance Research Center, University of Pittsburgh, \\ Pittsburgh, PA, USA
}

\begin{abstract}
Purpose-Instillation of novel contrast mixture (NCM) was recently shown to improve the contrast resolution of rat bladder wall with high contrast-to-noise ratio (CNR). Here, the clinical safety and the feasibility of NCM-enhanced MRI to achieve artifact-free visualization of human bladder wall suitable for quantitative measurement of the magnetic resonance (MR) longitudinal relaxation time (T1) was assessed.
\end{abstract}

\begin{abstract}
Methods-Six female subjects [two controls and two with Hunner-type interstitial cystitis IC and two with non-Hunner-type IC] consented for MRI at $3 \mathrm{~T}$ before and after instillation of NCM [4 $\mathrm{mM}$ gadobutrol and $5 \mathrm{mM}$ ferumoxytol in $50 \mathrm{~mL}$ of sterile water for injection]. Single breath-hold fast MR acquisition in large readout bandwidth for 5-mm-thick single slice with variable flip angle was applied to minimize the motion and chemical shift artifacts in measurements of bladder wall thickness (BWT), CNR and T1 from 20 pixels.
\end{abstract}

Results-NCM instillation in subjects did not evoke pain or discomfort. Fourfold increase in bladder wall CNR $(* p<0.02)$ and pixel size of $0.35 \mathrm{~mm}$ with minimal influence of artifacts allowed accurate determination of bladder wall thinning $\sim 0.46 \mathrm{~mm}$ from $50 \mathrm{~mL} \mathrm{NCM}\left({ }^{*} p<0.05\right)$. Pre-contrast bladder wall T1 of $1544 \pm 34.2 \mathrm{~ms}$ was shortened to $860.09 \pm 13.95 \mathrm{~ms}$ in Hunnertype IC $(* p<0.0001)$ relative to only $1257.42 \pm 20.59$ and $1258.16 \pm 6.16 \mathrm{~ms}$ in non-Hunner-type IC and controls, respectively.

\footnotetext{
Correspondence to: Pradeep Tyagi.

Compliance with ethical standards

Conflict of interest Pradeep Tyagi, Chan-Hong Moon and Christopher Chermansky declare that they have no conflict of interest. Joseph Janicki and Jonathan Kaufman are the employees of Lipella Pharmaceuticals, and Jonathan Kaufman is the inventor of the novel contrast mixture.

Ethical approval All procedures performed in studies involving human participants were in accordance with the Ethical Standards of the University of Pittsburgh Institutional Review Board and with the 1964 Helsinki Declaration and its later amendments or comparable ethical standards.

Informed consent Informed consent was obtained from all individual participants included in the study.
} 
Conclusion-Findings demonstrate the safety and feasibility of NCM-enhanced MRI to achieve artifact-free differential contrast and spatial resolution of human bladder wall, which is suitable for measuring BWT and pixel-wise measurement of T1 in post-contrast setting.

\section{Keywords}

Human bladder; MRI; T1 mapping; Gadolinium; Ferumoxytol

\section{Introduction}

Magnetic resonance imaging (MRI) is one of the preferred modalities for tomographic medical imaging of visceral organs [1]. Improved soft tissue contrast in three-dimensional anatomy without exposure to ionizing radiation [2] makes MRI a superior imaging technique over X-ray computed tomography (CT). Since there is no standard reference signal in MR image, the quality can be described by the contrast-to-noise ratio (CNR) and the spatial resolution per image pixel [3].

The CNR of the conventional T1-weighted (T1w) and T2-weighted (T2w) MRI image relies on the differences of adjacent tissues with regard to longitudinal relaxation time (T1) [4] and transverse relaxation time (T2) $[1,5]$, respectively. Human bladder wall is more distinguishable in T2w images, but anatomic [6] and functional details such as bladder wall thickness (BWT) are more accurately represented in T1w image [7]. BWT is a recognized indicator of pathological changes in bladder wall [8]. T1 of any tissue [9] is a first-order time constant which represents the time taken by its protons' spins to realign with the external magnetic field following excitation by radiofrequency pulse [10]. Body structures with short $\mathrm{T} 1$ such as perivesical fat $(\sim 0.2 \mathrm{~s})$ appear brighter than the structures with longer $\mathrm{T} 1$ such as urine and bladder wall ( $\sim \mathrm{s})$ on T1w images [4]. Recent advances allow quantitative measurement of T1 in tissues [11] to, serve as an objective and reproducible parameter for noninvasive characterization of normal and pathological changes [10].

Yet, the long T1 value of the bladder wall results in difficulty with quantitative T1 measurement using conventional MRI [4]. To acquire high MR signal of long T1 tissues with $\mathrm{T} 1 \mathrm{w}$ pulse sequence, the repetition time (TR) needs to be 3-5 times longer than the T1 relaxation time. Therefore, long T1 value $(\sim 1 \mathrm{~s})$ of bladder wall necessitates TR longer than $2 \mathrm{~s}$, which leads to lower spatial resolution [12]. Also, multiple signal averages with long TR prolong the image acquisition period (> $5 \mathrm{~min}$ ) [5], and this increases the susceptibility to artifacts from involuntary motion (respiration and bowel peristalsis). Furthermore, thin human bladder wall ( $3 \mathrm{~mm})$ [13] consists of muscle and fat tissue components in different layers that displace the respective MR signals in the readout direction. This leads to chemical shift mis-registration of the perivesical fat signal which further exaggerates motion artifacts [14]. Chemical shift artifact refers to the signal alterations that result from the 440$\mathrm{Hz}$ differences in the resonant frequencies of fat and water protons at $3 \mathrm{~T}[14,15]$, and this can be mitigated by high bandwidth per pixel in readout. Another factor responsible for poorly differentiated bladder wall in conventional T1w image is the long T1 value [4] of the urine present within the bladder lumen. 
Long $\mathrm{T} 1$ value of bladder wall can be shortened by the intravenous injection [2] or instillation $[16,17]$ of gadolinium-based contrast agents (GBCA); however, the CNR of bladder wall is not reliably increased with the injection or instillation of a single contrast agent GBCA for the following reasons. Injected GBCA initially diffuses into the extracellular volume (ECV) [10] of the lamina propria, but the urinary excretion of the injected GBCA [18] also brings it into the lumen and the consequent shortening of T1 with contrast in both the lumen and bladder wall makes them indistinguishable $[16,17]$ from the short T1 value of perivesical fat. Moreover, contrast enhancement with GBCA is based on shortening of both $\mathrm{T} 1$ and $\mathrm{T} 2$ relaxation time constants of adjacent water protons [19], which makes the signal intensity changes of thin bladder wall in post-contrast images [16] prone to the confounding influences from T2 $[20,21]$ or proton density. Such confounding influences $[20,21]$ have lesser impact on the quantitative measurement of the bladder wall T1, as proposed here.

We recently published that instillation of a novel contrast mixture (NCM) improves the rat bladder wall CNR at the 7-T animal scanner [18]. NCM is a defined mixture of paramagnetic GBCA and the superparamagnetic iron oxide (SPIO) composed of different sized particles and having different contrast effects on spin-echo MRI (Fig. 1). The present study seeks to test the clinical safety and the feasibility of NCM-enhanced MRI at 3-T scanner.

\section{Methods}

\section{Subjects}

Since primary outcome was feasibility and not a health-care outcome, the study was exempted from registration as a clinical trial and the study protocol was approved by the Institutional Review Board of University of Pittsburgh (PRO15070052). Total of six female subjects (25-78 years old) were recruited and all consented to the study, which includes four IC/BPS [two Hunner-type IC/BPS and two non-Hunner-type IC/BPS] and two asymptomatic healthy controls. Diagnosis of IC/BPS was established as per the SUFU definition, a significant pelvic pain in conjunction with urinary urgency and frequency without an anatomic cause. Enrolled IC/BPS patients completed the Interstitial Cystitis Symptoms Index and Interstitial Cystitis Problem Index questionnaire with scores above 6 and 8 , respectively. Hunner's lesions were confirmed by prior cystoscopic examination, and none of the patients had recently been hydrodistended nor had received any intravesical therapy.

\section{MRI}

The studies were conducted at 3-T clinic scanner with $60 \mathrm{~cm}$ wide bore size, $45 \mathrm{mT} / \mathrm{m}$ maximum gradient amplitude and a slew rate of $200 \mathrm{~T} / \mathrm{m} / \mathrm{s}$ with a $100 \%$ duty cycle (Siemens AG, Erlangen, Germany). Subjects lied in a supine position, and respiratory monitoring belt was placed under the receiver coil for the duration of imaging period for checking the respiration status and the breath hold during study. Body transmit coil was used for radiofrequency (RF) transmission, and a 4-channel flexible receiver RF coil with high sensitivity to the bladder was applied for signal reception. The anatomic position of the 
bladder was first confirmed by performing a single-shot $\mathrm{T} 2 \mathrm{w}$ imaging via true fast imaging with steady precession sequence using TR and echo time (TE) of 6.94 and $3.06 \mathrm{~ms}$, respectively, during a breath hold.

Subsequently, two-dimensional (2D) fast low-angle shot (FLASH) sequence at variable flip angles (FA) of $3^{\circ}-23^{\circ}$ was performed with the following imaging parameters, TR/ TE of $5.5 / 2 \mathrm{~ms}$, field of view (FOV) of $180 \times 180 \mathrm{~mm}^{2}$, imaging matrix of $256 \times 256$ and 10 averaging. Single slice image of $5 \mathrm{~mm}$ thickness and $0.35-\mathrm{mm}$ in-plane resolution (after $2 \times$ interpolation) was acquired during a single breath hold of ( $17 \mathrm{~s})$ for each FA. After acquiring pre-contrast images, subjects were catheterized with lubricated 16-Fr coude or straight-tipped catheter in the supine position on the scanner table to remove the urine prior to $50 \mathrm{~mL}$ NCM instillation. NCM was compounded just before the instillation by mixing the GBCA gadobutrol (Gadovist, $1 \mathrm{mmol} / \mathrm{mL}$; Bayer Healthcare, Wayne, NJ) diluted 1:250 and ferumoxytol (Feraheme; $30 \mathrm{mg}$ SPIO/mL; AMAG Pharmaceuticals Inc., Waltham, MA) diluted 1:104 to a final concentration of 4 and $5 \mathrm{mM}$, respectively. Subsequently, subjects were repositioned to the position for pre-contrast scans and FLASH images at variable FA were repeated. Subjects were administered a single ciprofloxacin dose at the end of the imaging as a prophylaxis against infection from the instillation procedure.

\section{Data analysis}

Pre-contrast and post-contrast images were processed offline in Digital Imaging and Communications in Medicine (DICOM) format for measuring CNR, BWT and T1. CNR was calculated from the mean signal intensity (MSI) measured in regions of interest (ROI) in the bladder lumen versus bladder wall using noise reference of air, i.e., background region in MR image. ROIs were drawn in the region of maximum enhancement and included at least 20 pixels, chosen away from the respective border or edges so that slight errors and partial volume artifacts would not bias the final result [3]. For the pixel-based T1 mapping, signal intensities $(S)$ at FA $(a)$ of $4^{\circ}-15^{\circ}$ were fitted to the Ernst equation $S=\frac{M_{0} * \sin (\alpha) *(1-E 1)}{(1-E 1) * \cos (\alpha)}$ where $E 1=\exp (-\mathrm{TR} / \mathrm{T} 1), \mathrm{TR}$ is the repetition time, and $M_{0}$ is the equilibrium magnetization. T1 longitudinal relaxation time was estimated via parameter optimization, which was performed using SciPy Python package (https://www.scipy.org).

\section{Statistics}

Values are expressed as mean \pm standard deviation. Significance between pre- and postcontrast images was assessed by paired Student's $t$ test, and differences between the postcontrast values of individual subjects were assessed by two-way analysis of variance (ANOVA) followed by Tukey's test.

\section{Results}

\section{MR images of the phantoms}

Phantoms containing either water, GBCA (gadobutrol $4 \mathrm{mM}$ ), Fe (ferumoxytol $5 \mathrm{mM}$ ) and the NCM [composed of Fe $5 \mathrm{mM}$ and GBCA $4 \mathrm{mM}$ ] were scanned together at $3 \mathrm{~T}$ to analyze the effect of FA on the T1w signal intensity measured in arbitrary units (Fig. 2). The 
signal intensity of GBCA phantom increased from 275 to 614 to 792 at the corresponding FA of $6^{\circ}, 15^{\circ}$ and $23^{\circ}$ (Fig. 2a-c). Since signal intensity of GBCA phantom doubled from FA of $6^{\circ}-15^{\circ}, \mathrm{FAs} \leq 15^{\circ}$ were then used for human bladder image analysis. In comparison, the signal intensity of the NCM phantom only increased from 108 to 383 in images acquired from FA of $6^{\circ}-23^{\circ}$. Indifference in the signal intensity of the NCM and Fe phantoms demonstrates that contrast enhancement by GBCA in the NCM phantom is quenched by ferumoxytol [22] and the observed quenching of GBCA is independent of the FA. However, the quenching of GBCA in NCM is dependent on the proximity of GBCA to ferumoxytol, as evident from the bright bladder wall of rat bladder phantom (Fig. 1b) instilled with NCM 0.3 $\mathrm{mL}$. Hence, quenching of GBCA only occurs in lumen and the partitioning of GBCA away from lumen into the bladder wall results in contrast enhancement (Fig. 1b) [18].

\section{Pre-contrast MRI of human bladder}

Representative pre-contrast T1w and T2w images of human bladder are shown in Fig. 3. The visualization of the bladder wall in $\mathrm{T} 2 \mathrm{w}$ images (Fig. 3b, d) was superior to the pre-contrast T1w images (Fig. 3a, c), because bladder wall T1 is 259 times longer than the TR of $5.5 \mathrm{~ms}$ used for acquiring T1w images by FLASH sequence. T1w images achieved in-plane resolution of $0.35 \mathrm{~mm}^{2}$ and relatively high readout bandwidth of $574 \mathrm{~Hz} /$ pixel for T1w images ensures that the mis-mapping of the water and fat signals is limited within a pixel (i.e., 0.7 pixels $=440 \mathrm{~Hz} / 574 \mathrm{~Hz}$ ) $[14,15]$. The BWT measured with T1w and T2w images at equal bladder distension in the dome and lateral wall of six subjects was $3.39 \pm 0.74$ and $3.01 \pm 0.82 \mathrm{~mm}$, respectively. Slight underestimation of BWT in the T2w image is also reported from other groups [5], which is considered to be caused by the increased signal intensity of the urothelium and lamina propria relative to the detrusor smooth muscle [6].

\section{Post-contrast MRI of human bladder}

Compared to the pre-contrast T1w images (Fig. 4a, c, e) acquired with the same imaging parameters at the FA of $14^{\circ}$, a fourfold rise in CNR (57.84 \pm 32.01 vs. $12.34 \pm 9.63 ; * p<$ $0.02 ; n=6$, paired Student's $t$ test) was observed in post-contrast images acquired after NCM instillation (Fig. 4b, d, f). Images at different FA were serially acquired, and the image at FA $14^{\circ}$ was acquired 20 min after NCM instillation. High CNR between hypointense human bladder wall and the hyperintense lumen recapitulated the high CNR seen in rat bladder phantom, but the corresponding regions of hyperintensity and hypointensity were inversed (Fig. 1b). High CNR allowed us to quantify the significant bladder wall thinning from $3.39 \pm 0.74$ to $2.93 \pm 0.8 \mathrm{~mm}\left({ }^{*} p<0.05\right.$; paired Student's $t$ test) in post-contrast images. Although catheterization process caused mild discomfort in all subjects, none of the subjects reported any additional pain or discomfort from the instillation of NCM and holding NCM in bladder for $30 \mathrm{~min}$. Bladder wall of Hunner-type IC patients is certainly enhanced more than those of controls, and further analysis of the differences in $\mathrm{T} 1$ contrast for different subjects was performed as described below.

\section{T1 measurement}

The approach previously reported for the heart by Coolen et al. [23] was slightly modified for calculating T1 values at each pixel representing bladder wall in color-coded T1 map (Fig. 5). The pixels representing the pre-contrast bladder wall are of the same green to yellow 
color in all the six subjects (Fig. 5a, c, e), and the T1 value for the corresponding color in the color bar allowed us to calculate a mean value of $1544 \pm 34.2 \mathrm{~ms}(n=6)$. In comparison, color-coded T1 map of post-contrast bladder wall depicts a color gradient (Fig. 5b, d, f), which indicates a greater $\mathrm{T} 1$ shortening from GBCA diffusion in pixels representing the inner layer (urothelium) (dark blue color) > pixels representing the middle-layer lamina propria (blue to green) > pixel representing the outer detrusor layer (green to yellow). Larger blue- to green-colored band of pixels in bladder wall of Hunner-type IC patients indicates an increased diffusion of GBCA [10, 24] compared to controls and non-Hunner-type IC/BPS patients. Differences in color-coded T1 map (Fig. 5b, d, f) of controls and Hunner-type IC patients confirm that confounding influences [21] mask the differences in T1 contrast (Fig. 4b, d, f). Color-coded T1 map for post-contrast images indicated a GBCA-induced shortening of $\mathrm{T} 1$ from the mean pre-contrast value of $1544 \pm 34.2 \mathrm{~ms}$. Comparison of postcontrast T1 values for individual subjects revealed that T1 shortened to $860.09 \pm 13.95 \mathrm{~ms}$ in Hunner-type IC, which was nearly double of the T1 shortening noted in non-Hunner-type IC and controls with $1257.42 \pm 20.59$ and $1258.16 \pm 6.16 \mathrm{~ms}$, respectively $\left({ }^{*} p<0.0001\right.$, twoway ANOVA followed by Tukey's test; Fig. 6).

\section{Discussion}

Overall, published findings on NCM-enhanced MRI of rat bladder wall [18] were successfully translated to the clinical setting with the acquisition of artifact-free, highresolution images of human bladder wall following $50 \mathrm{~mL}$ instillation of NCM. Past attempts at contrast-enhanced MRI using GBCA as a single agent were not successful in acquiring artifact-free images of high spatial resolution [16, 17, 25], which is critical for quantitative T1 measurement.

NCM-enhanced MRI, using a mixture of two contrast agents (GBCA and ferumoxytol) allowed accurate delineation of the inner and outer boundaries of bladder wall with an increased differential contrast from lumen. Smaller pixel size $\left(0.35 \times 0.35 \mathrm{~mm}^{2}\right)$ and increased number of pixels representing the $\sim 3$-mm-thick human bladder wall drastically increased the sensitivity of NCM-enhanced MRI to measure the significant bladder wall thinning from $50 \mathrm{~mL}$ instillation of NCM. Bladder dome and lateral wall are recommended for the measurement of BWT [8], and the values reported for those regions [13] were successfully measured. Since BWT is underestimated in T2w images [6], accurate measurement of BWT described here by NCM-enhanced T1w MRI following $50 \mathrm{~mL}$ instillation is clinically relevant for the accurate determination of bladder wall hypertrophy secondary to either IC/BPS, BPH-mediated outlet obstruction or aging.

Moreover, $50 \mathrm{~mL}$ is the standard volume for intravesical therapies of IC/PBS patients [26], which portends an improved tolerance of NCM-enhanced MRI by IC/BPS patients with low bladder capacity between 200 and $400 \mathrm{~mL}$ [27]. In a recently reported study, Towner et al. [16] instilled GBCA as a single contrast agent in IC/BPS patients, but the volume of instillation was significantly larger than $50 \mathrm{~mL}$. Considering the change in BWT with the instillation of $50 \mathrm{~mL}$, the bladder wall distension from large-volume GBCA instillation [16, 25 ] is likely to hinder the differentiation of the tissue layers and may provoke involuntary motion in sensitive patients. Since instillation of GBCA as a single agent shortens T1 in both 
lumen and bladder wall [25], the resulting low CNR [21] also hampers BWT measurement. CNR is also influenced by the chemical shift artifact [18], which appears as regional differences in the brightness of the bladder wall [4] in the frequency-encoding direction [4] that extends further at the lower receiver frequency bandwidth [18].

NCM-enhanced MRI technique developed here affords smaller pixel size and higher bandwidth $574 \mathrm{~Hz}$ per pixel, which attenuates the chemical shift mis-registration of the perivesical fat signal and achieves visual separation between lumen and bladder wall that is typical of T2w images [5] without compromising the accuracy in BWT measurement. The intravesical concentration of $4 \mathrm{mM}$ for GBCA in NCM was optimized in previous studies $[17,18]$, which is nearly double of the peak plasma concentration [28] that reaches lamina propria immediately after intravenous injection of GBCA [2]. The 1:250 dilution of GBCA used in NCM is lower than the concentration instilled as a single agent in bladder cancer [25], and IC patients [16], but higher than the dilution used in vesicoureteral reflux [17].

FLASH sequence used here for assessing the contrast enhancement of bladder wall at $3 \mathrm{~T}$ was previously used after intradetrusor injection of GBCA in OAB patients at 1.5 T [29]. Human bladder wall is visualized clearly in the post-contrast FLASH images, but not in the pre-contrast FLASH images, because the TR of 4-5.5 ms [29] is > 280 times shorter than the bladder wall T1. However, short TR of $5.5 \mathrm{~ms}$ allowed fast acquisition of 5-mm-thick single slice over a single breath hold of $\sim 17 \mathrm{~s}$ at each FA, which reduces the susceptibility to motion artifacts [5]. Short TR also improved the spatial resolution with $>2$-fold reduction in the size of pixels from $0.9 \mathrm{~mm}$ [12] to $0.35 \mathrm{~mm}$. Constant TR at different FA helped us achieve stable steady-state conditions for obtaining T1 maps from radiofrequency-spoiled gradient-echo data sets as the signal intensity then becomes a function of the tissue T1 [23, 30].

Pre-contrast human bladder wall $\mathrm{T} 1$ of $1544 \pm 34.2 \mathrm{~ms}$ at $3 \mathrm{~T}$ agrees with the values predicted from measurements at lower field strength of $0.3 \mathrm{~T}$ [4] and $1.5 \mathrm{~T} \mathrm{[31].} \mathrm{T1}$ shortening in post-contrast images [24] is consistent with the diffusion of GBCA into the bladder wall from instilled NCM in lumen [18] and is a more reliable [20,21] measure of bladder wall permeability than the probability distribution of bladder wall signal intensity in post-contrast images [16]. Since GBCA is known to remain in the ECV [10], a greater T1 shortening in pixels representing the urothelium and lamina propria of bladder wall is consistent with the higher proportion of ECV in urothelium and lamina propria compared to the detrusor smooth muscle region [6]. Hence, we propose that pixel-wise T1 mapping performed after NCM instillation can allow differentiation of the bladder wall into different tissue layers with an increased depth of GBCA diffusion in the Hunner-type IC/BPS patients.

This pilot study was limited by the small size and was not powered to demonstrate the group-wise differences between the controls and non-Hunner-type IC/BPS subjects for BWT and $\mathrm{T} 1$ value. Nonetheless, pixel-wise T1 measurement could provide data from 20 pixels to compare the differences in post-contrast $\mathrm{T} 1$ values of individual subjects. The results of significantly shortened bladder wall T1 in two Hunner-type IC/BPS patients are promising and warrant further evaluation in an independent trial with a larger sample size. Whether the 
shortening of bladder wall $\mathrm{T} 1$ in post-contrast images of Hunner-type IC/BPS patients is caused by an increase in interstitial space can be verified by histopathologic confirmation in future studies. Histopathologic confirmation will demonstrate the potential of T1 mapping [11] to inform about the interstitial space in bladder wall without removing the bladder wall biopsy. NCM-enhanced MRI described here coupled with the tonicity measurements [1] also has the potential to identify bladder-centric manifestation from other manifestations of IC/BPS patients.

\section{Conclusion}

The safety and feasibility of NCM-enhanced MRI to achieve artifact-free differential contrast (> 4-fold higher CNR) and spatial resolution of human bladder wall recapitulates the published preclinical findings in the clinical setting. Accurate measurement of BWT and pixel-wise measurement of bladder wall $\mathrm{T} 1$ in post-contrast setting hold promise in the noninvasive characterization of diffuse interstitial space in bladder wall for improved diagnosis and clinical care of IC/ BPS patients.

\section{Acknowledgments}

Funding This project was funded by NIDDK Grant 1R41DK108397. Authors would also like to acknowledge the contribution of Janet Erick-son and Anna Wecht in patient recruitment and for assistance during scanning.

\section{References}

1. Ackerman AL, Lee UJ, Jellison FC, Tan N, Patel M, Raman SS, Rodriguez LV. MRI suggests increased tonicity of the levator ani in women with interstitial cystitis/bladder pain syndrome. Int Urogynecol J. 2016; 27(1):77-83. https://doi.org/10.1007/s00192-015-2794-6. [PubMed: 26231233]

2. Rabie E, Faeghi F, Izadpanahi MH, Dayani MA. Role of dynamic contrast-enhanced magnetic resonance imaging in staging of bladder cancer. J Clin Diagn Res JCDR. 2016; 10(4):01-05. https:// doi.org/10.7860/jcdr/2016/17596.7690.

3. Magnotta VA, Friedman L, First B. Measurement of signal-to-noise and contrast-to-noise in the fBIRN multicenter imaging study. J Digit Imaging. 2006; 19(2):140-147. https://doi.org/10.1007/ s10278-006-0264-x. [PubMed: 16598643]

4. Fisher MR, Hricak H, Crooks LE. Urinary bladder MR imaging. Part I. Normal and benign conditions. Radiology. 1985; 157(2):467-470. https://doi.org/10.1148/radiology.157.2.4048457. [PubMed: 4048457]

5. Ma Z, Jorge RN, Mascarenhas T, Tavares JM. Novel approach to segment the inner and outer boundaries of the bladder wall in T2-weighted magnetic resonance images. Ann Biomed Eng. 2011; 39(8):2287-2297. https://doi.org/10.1007/s10439-011-0324-3. [PubMed: 21559984]

6. Takeda K, Kawaguchi T, Shiraishi T, Kobayashi S, Hayashi N, Yanagawa M, Tochigi H, Sakuma H, Kawamura J, Nakagawa T. Normal bladder wall morphology in Gd-DTPA-enhanced clinical MR imaging using an endorectal surface coil and histological assessment of submucosal linear enhancement using [14C] Gd-DOTA autoradiography in an animal model. Eur J Radiol. 1998; 26(3):290-296. [PubMed: 9587759]

7. Alsinnawi M, Torreggiani W, Sheikh M, Thomas A, Donnellan J, Flynn R, McDermott TE, Thornhill J. Delayed contrast-enhanced MRI to localize Botox after cystoscopic intravesical injection. Int Urol Nephrol. 2015; 47(6):893-898. https://doi.org/10.1007/s11255-015-0976-2. [PubMed: 25894961]

8. Oelke M, Khullar V, Wijkstra H. Review on ultrasound measurement of bladder or detrusor wall thickness in women: techniques, diagnostic utility, and use in clinical trials. World J Urol. 2013; 31(5):1093-1104. https://doi.org/10.1007/s00345-013-1030-6. [PubMed: 23386057] 
9. Bloch F. The principle of nuclear induction. Science. 1953; 118(3068):425-430. https://doi.org/ 10.1126/science.118.3068.425. [PubMed: 17847222]

10. Arheden H, Saeed M, Higgins CB, Gao DW, Bremerich J, Wyttenbach R, Dae MW, Wendland MF. Measurement of the distribution volume of gadopentetate dimeglumine at echo-planar MR imaging to quantify myocardial infarction: comparison with 99mTc-DTPA autoradiography in rats. Radiology. 1999; 211(3):698-708. https://doi.org/10.1148/radiology.211.3.r99jn41698. [PubMed: 10352594]

11. Stadler A, Jakob PM, Griswold M, Stiebellehner L, Barth M, Bankier AA. T1 mapping of the entire lung parenchyma: influence of respiratory phase and correlation to lung function test results in patients with diffuse lung disease. Magn Reson Med. 2008; 59(1):96-101. https://doi.org/ 10.1002/mrm.21446. [PubMed: 18098282]

12. Maeda H, Kinukawa T, Hattori R, Toyooka N, Furukawa T, Kuhara H. Detection of muscle layer invasion with sub-millimeter pixel MR images: staging of bladder carcinoma. Magn Reson Imaging. 1995; 13(1):9-19. [PubMed: 7898285]

13. Lammle M, Beer A, Settles M, Hannig C, Schwaibold H, Drews C. Reliability of MR imagingbased virtual cystoscopy in the diagnosis of cancer of the urinary bladder. AJR Am J Roentgenol. 2002; 178(6):1483-1488. https://doi.org/10.2214/ajr.178.6.1781483. [PubMed: 12034625]

14. Hood MN, Ho VB, Smirniotopoulos JG, Szumowski J. Chemical shift: the artifact and clinical tool revisited. Radiographics. 1999; 19(2):357-371. https://doi.org/10.1148/radiographics. 19.2.g99mr07357. [PubMed: 10194784]

15. Verma S, Rajesh A, Prasad SR, Gaitonde K, Lall CG, Mouraviev V, Aeron G, Bracken RB, Sandrasegaran K. Urinary bladder cancer: role of MR imaging. Radiographics. 2012; 32(2):371387. https://doi.org/10.1148/rg.322115125. [PubMed: 22411938]

16. Towner RA, Wisniewski AB, Wu DH, Van Gordon SB, Smith N, North JC, McElhaney R, Aston CE, Shobeiri SA, Kropp BP, Greenwood-Van Meerveld B, Hurst RE. A feasibility study to determine whether clinical contrast enhanced magnetic resonance imaging can detect increased bladder permeability in patients with interstitial cystitis. J Urol. 2016; 195(3):631-638. https:// doi.org/10.1016/j.juro.2015.08.077. [PubMed: 26307161]

17. Lee SK, Chang Y, Park NH, Kim YH, Woo S. Magnetic resonance voiding cystography in the diagnosis of vesicoureteral reflux: comparative study with voiding cystourethrography. J Magn Reson Imaging JMRI. 2005; 21(4):406-414. https://doi.org/10.1002/jmri.20273. [PubMed: 15779038]

18. Tyagi P, Janicki JJ, Hitchens TK, Foley LM, Kashyap M, Yoshhimura N, Kaufman J. Novel contrast mixture improves bladder wall contrast for visualizing bladder injury. Am J Physiol Renal Physiol. 2017; 313(2):F155-F162. https://doi.org/10.1152/ajprenal.00609.2016. [PubMed: 28356290]

19. Johnson GA, Cofer GP, Gewalt SL, Hedlund LW. Morphologic phenotyping with MR microscopy: the visible mouse. Radiology. 2002; 222(3):789-793. https://doi.org/10.1148/radiol.2223010531. [PubMed: 11867802]

20. Xie L, Layton AT, Wang N, Larson PE, Zhang JL, Lee VS, Liu C, Johnson GA. Dynamic contrastenhanced quantitative susceptibility mapping with ultrashort echo time MRI for evaluating renal function. Am J Physiol Renal Physiol. 2016; 310(2):F174-F182. https://doi.org/10.1152/ajprenal. 00351.2015. [PubMed: 26447222]

21. Elster AD, Sobol WT, Hinson WH. Pseudolayering of Gd-DTPA in the urinary bladder. Radiology. 1990; 174(2):379-381. https://doi.org/10.1148/radiology.174.2.2296649. [PubMed: 2296649]

22. Beyersdorff D, Taupitz M, Giessing M, Turk I, Schnorr D, Loening S, Hamm B. The staging of bladder tumors in MRT: the value of the intravesical application of an iron oxide-containing contrast medium in combination with high-resolution T2-weighted imaging. RoFo Fortschritte auf dem Gebiete der Rontgenstrahlen und der Nuklearmedizin. 2000; 172(6):504-508. https://doi.org/ 10.1055/s-2000-3751. [PubMed: 10916545]

23. Coolen BF, Geelen T, Paulis LE, Nauerth A, Nicolay K, Strijkers GJ. Three-dimensional T1 mapping of the mouse heart using variable flip angle steady-state MR imaging. NMR Biomed. 2011; 24(2):154-162. https://doi.org/10.1002/nbm.1566. [PubMed: 20960583] 
24. Gowland P, Mansfield P, Bullock P, Stehling M, Worthington B, Firth J. Dynamic studies of gadolinium uptake in brain tumors using inversion-recovery echo-planar imaging. Magn Reson Med. 1992; 26(2):241-258. [PubMed: 1513249]

25. Sparenberg A, Hamm B, Hammerer P, Samberger V, Wolf KJ. The diagnosis of bladder carcinomas by NMR tomography: an improvement with Gd-DTPA? RoFo Fortschritte auf dem Gebiete der Rontgenstrahlen und der Nuklearmedizin. 1991; 155(2):117-122. https://doi.org/10.1055/ s-2008-1033231. [PubMed: 1878537]

26. Rawls WF, Cox L, Rovner ES. Dimethyl sulfoxide (DMSO) as intravesical therapy for interstitial cystitis/bladder pain syndrome: a review. Neurourol Urodyn. 2017; 36(7):1677-1684. https:// doi.org/10.1002/nau.23204. [PubMed: 28220525]

27. Walker, SJ., Zambon, J., Andersson, KE., Langefeld, C., Matthews, CA., Badlani, G., Bowman, H., Evans, RJ. Bladder capacity is a biomarker for a bladder-centric versus systemic manifestation in interstitial cystitis/bladder pain syndrome. J Urol. 2017. https://doi.org/10.1016/j.juro.2017.02.022

28. Tiderius C, Hori M, Williams A, Sharma L, Prasad PV, Finnell M, McKenzie C, Burstein D. dGEMRIC as a function of BMI. Osteoarthr Cartil. 2006; 14(11):1091-1097. https://doi.org/ 10.1016/j.joca.2006.05.007. [PubMed: 16782361]

29. Mehnert U, Boy S, Schmid M, Reitz A, von Hessling A, Hodler J, Schurch B. A morphological evaluation of botulinum neurotoxin A injections into the detrusor muscle using magnetic resonance imaging. World J Urol. 2009; 27(3):397-403. https://doi.org/10.1007/ s00345-008-0362-0. [PubMed: 19145439]

30. Deoni SC, Rutt BK, Peters TM. Rapid combined T1 and T2 mapping using gradient recalled acquisition in the steady state. Magn Reson Med. 2003; 49(3):515-526. https://doi.org/10.1002/ mrm.10407. [PubMed: 12594755]

31. Kanazawa Y, Miyati T, Sato O. Hemodynamic analysis of bladder tumors using T1-dynamic contrast-enhanced fast spin-echo MRI. Eur J Radiol. 2012; 81(8):1682-1687. https://doi.org/ 10.1016/j.ejrad.2011.04.013. [PubMed: 21543177] 

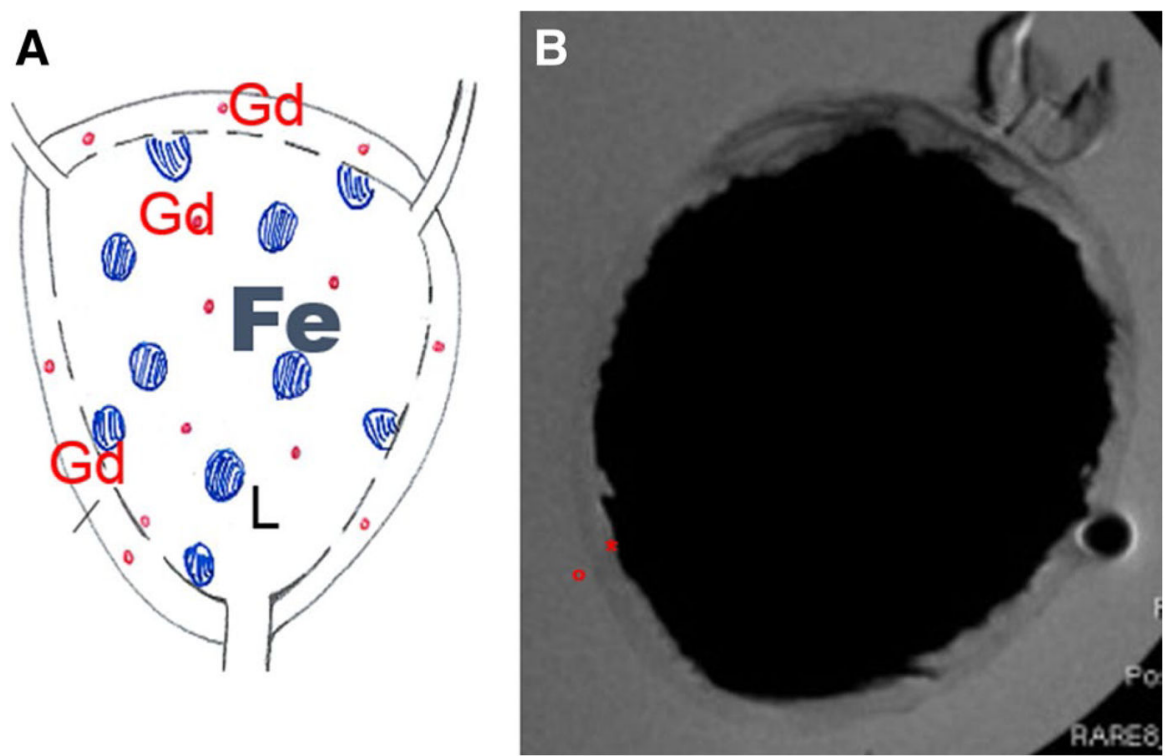

Fig. 1.

NCM-enhanced MRI relies on the differences in the particle size and contrast mechanisms of two FDA-approved agents for increasing the CNR of human bladder wall. a Schematic illustration depicting the diffusion of smaller-sized gadolinium-based contrast agent GBCA (Gd) molecules into the ECV of the bladder wall leads to shortened T1 (positive contrast), while large molecular size of ferumoxytol $(\mathrm{Fe})$ allows hypointensity to dominate within the lumen due to $\mathrm{T} 2$ relaxation (signal loss) in T1w images. b $\mathrm{T} 1 \mathrm{w}$ image acquired at $7 \mathrm{~T}$ (TR/TE 500/7.3 ms) of ex vivo rat bladder phantom instilled with $0.3 \mathrm{~mL}$ NCM. GBCA diffusion into the bladder wall from lumen increases the differential contrast of the hyperintense bladder wall with inner (asterisk) and outer border (open circle). Retention of ferumoxytol in lumen quenches the GBCA-mediated enhancement leading to the darkened lumen 

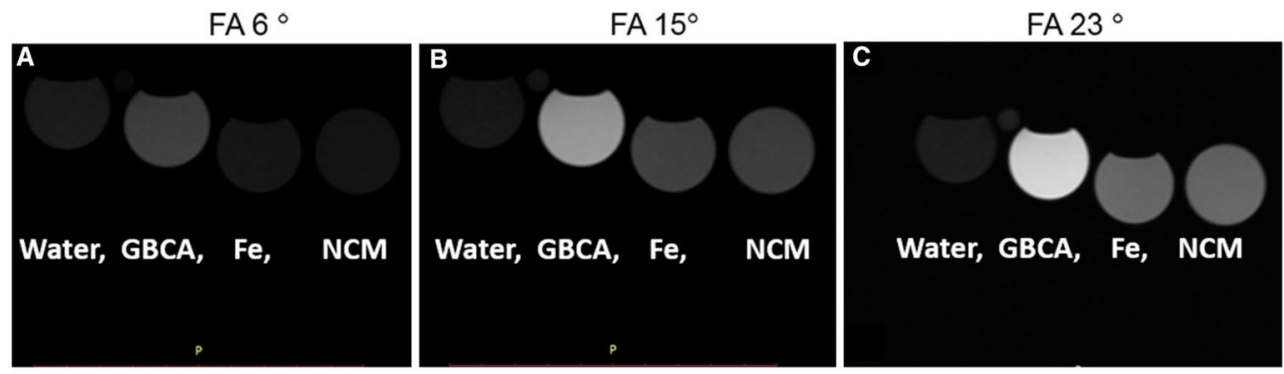

Fig. 2.

Effect of FA on T1w signal intensity at $3 \mathrm{~T}$ - the signal intensity (arbitrary units) of the GBCA phantom doubled in T1w 2D-FLASH image (b) acquired at FA of $15^{\circ}(614)$, compared to the image acquired at FA of $6^{\circ}$ (272) shown in (a). Since signal intensity of GBCA phantom doubled from FA of $6^{\circ}-15^{\circ}$, T1w 2D-FLASH image acquired at FAs of $\leq$ $15^{\circ}$ was used for measuring CNR, BWT and T1 of human subjects. Lack of difference in the signal intensity of the NCM and Fe phantoms demonstrates that contrast enhancement by GBCA in NCM phantom is quenched by ferumoxytol $(\mathrm{Fe})$ and the observed quenching of GBCA is independent of the FA. All four phantoms were imaged together at different FA with 2D-FLASH sequence (TR/TE 5/2 ms) at 3-T scanner 

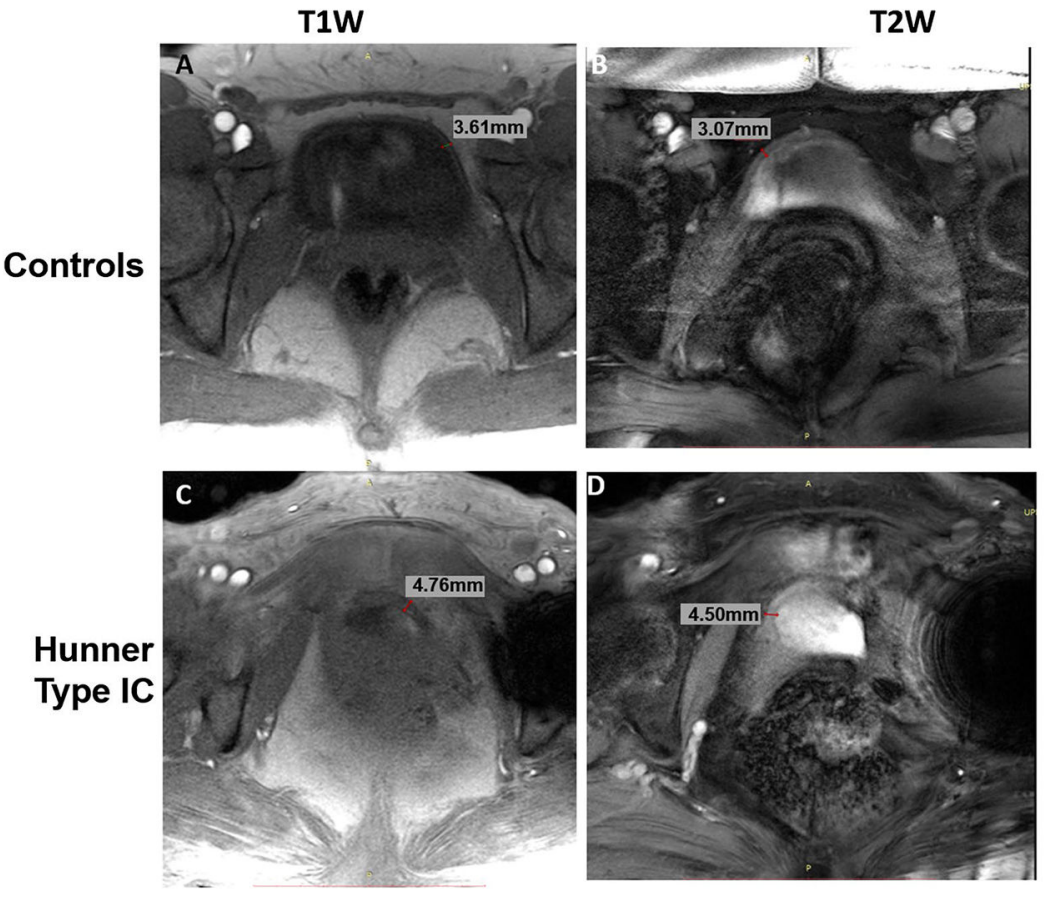

Fig. 3.

BWT is underestimated in T2w MRI. Representative pre-contrast T1w 2D-FLASH (TR/TE 5.5/2 ms) and T2w images (TR/ TE 6.94/3.06 ms) of the controls (a, b) and Hunner-type IC/BPS subjects (c, d). A slight underestimation of BWT by T2w is suggested by BWT measurements of $3.39 \pm 0.74$ versus $3.01 \pm 0.82 \mathrm{~mm}$ in $\mathrm{T} 1 \mathrm{w}$ and $\mathrm{T} 2 \mathrm{w}$ images, respectively $(n=6)$. Thickened bladder wall noted in the Hunner-type IC/BPS patient shown here (c, d) corroborates the increased circumferential thickness noted by other methods 


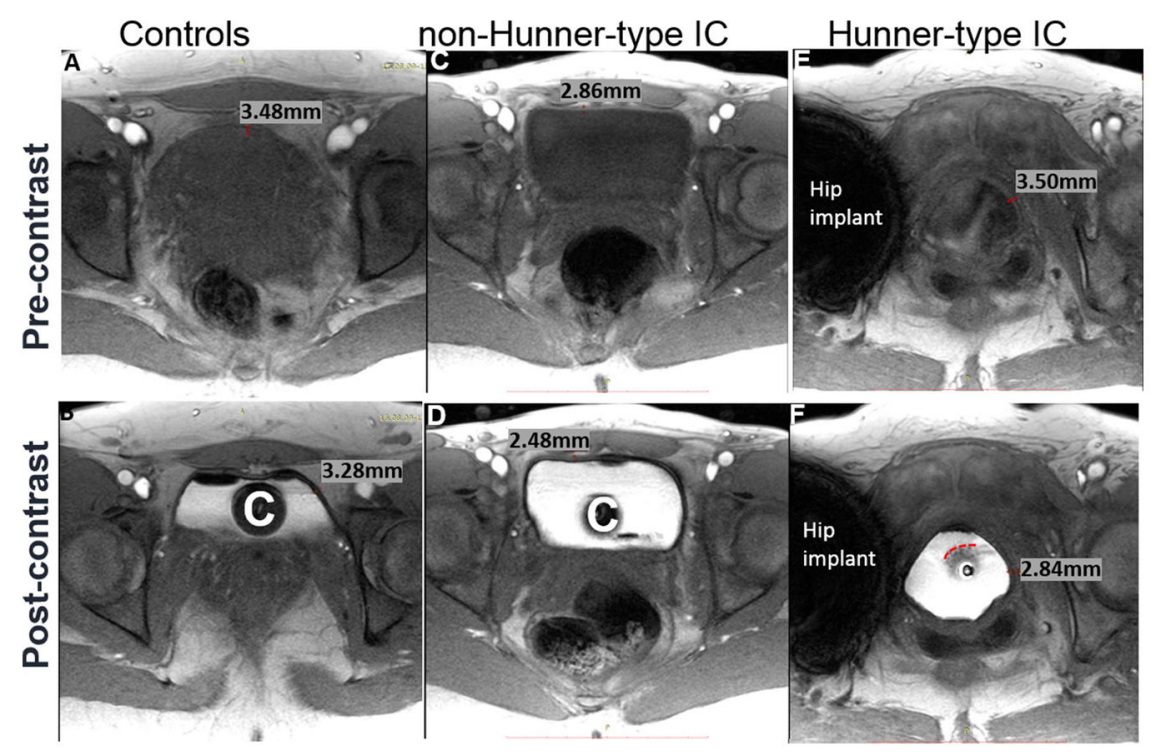

Fig. 4.

NCM-enhanced MRI improves the human bladder wall CNR. Representative pre- and postcontrast images of female control subjects, non-Hunner-type IC and Hunner-type IC patients acquired in orthogonal orientation at 3-T scanner are shown. NCM instillation raised the CNR of human bladder wall in post-contrast images (57.84 \pm 32.01 vs. $12.34 \pm 9.63$; * $p<$ 0.02, paired Student's $t$ test; $n=6)(\mathbf{b}, \mathbf{d}, \mathbf{f})$ by fourfold over the pre-contrast images $(\mathbf{a}, \mathbf{c}, \mathbf{e})$ acquired with the same parameters. Fast image acquisition over single breath hold of $\sim 17 \mathrm{~s}$ at the FA of $14^{\circ}$ with slice thickness of $5 \mathrm{~mm}$, matrix size $256 \times 256$, FOV of $18 \times 18 \mathrm{~cm}^{2}$ and 10 averaging achieved in-plane resolution of $0.35 \mathrm{~mm}$ for CNR measurement in 20 pixels. Increased differential contrast between lumen and bladder wall, smaller pixel size and large bandwidth $574 \mathrm{~Hz}$ per pixel allowed accurate determination of the significant bladder wall thinning from $3.39 \pm 0.74$ to $2.93 \pm 0.8 \mathrm{~mm}$ (paired Student's $t$ test, $* p<0.05$, $n=6$ ) in post-contrast images acquired after $50 \mathrm{~mL}$ instillation. (BWT is shown by the inset legend.) Trace amount of blood oozing from lesions in Hunner-type IC/BPS patient is shown by gray granules (indicated by red dotted line) against the bright background of lumen near the catheter shown by letter $\mathrm{C}$ in post-contrast images. The catheter is shown by the letter $\mathrm{C}$ in post-contrast images, and the dark areas within the lumen are caused by air bubble introduced from the instillation procedure. Titanium hip implant casts a dark shadow in pelvic region of Hunner-type IC patient, but the effect on bladder wall imaging was minimal 


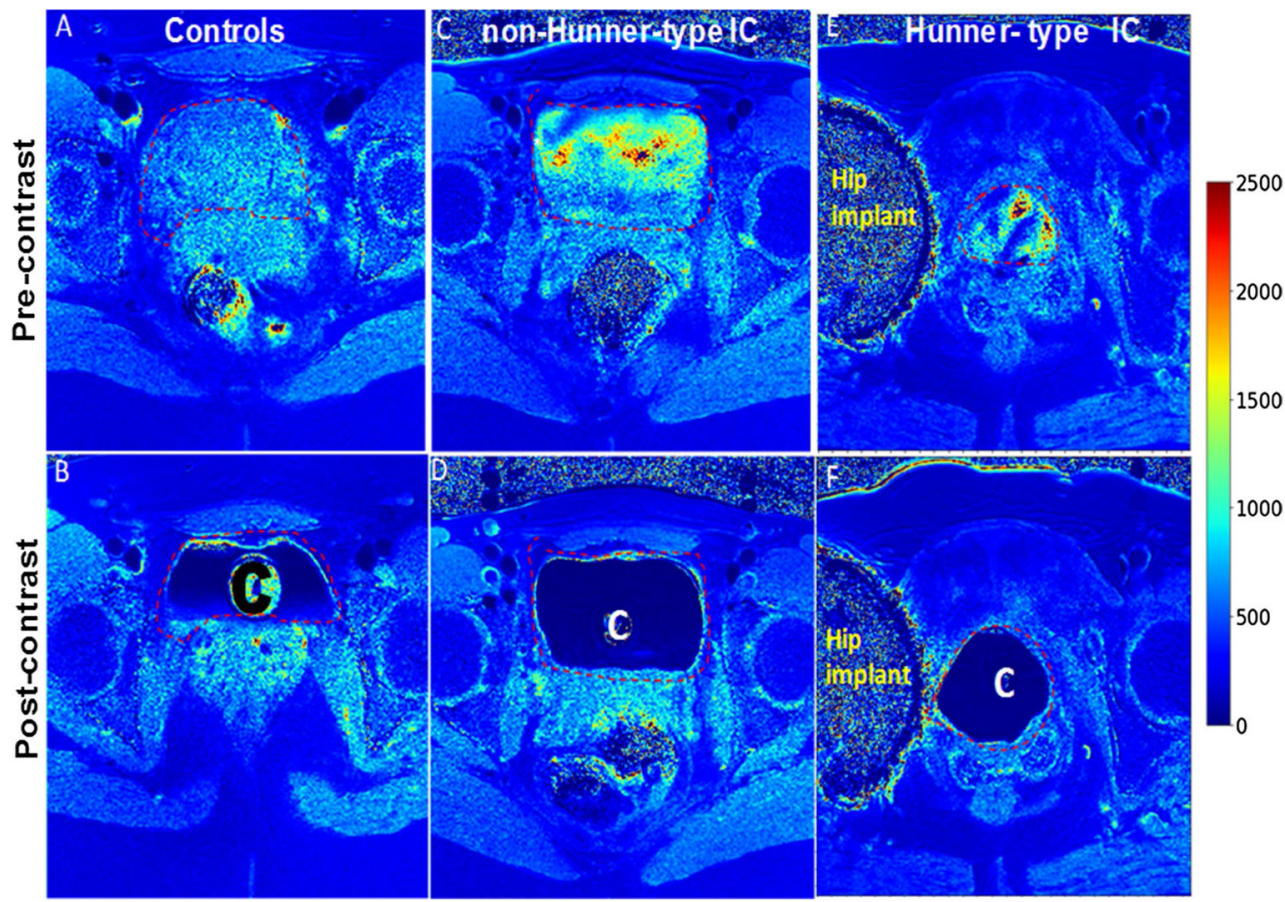

Fig. 5.

T1 measurement of human bladder wall $(\mathbf{a}-\mathbf{f})$ corresponds to respective images shown in Fig. 4. Calculated T1 values over the entire field of view are illustrated in a color-coded map, where dark blue-colored pixel assigns T1 of $0 \mathrm{~ms}$ and dark red-colored pixel assigns $\mathrm{T} 1$ of $2500 \mathrm{~ms}$. a, c, e The pixels representing bladder wall (within the red dotted line regions in all figures) in pre-contrast images of six subjects are in the range of green to yellow, which together generate a mean value of $1544 \pm 34.2 \mathrm{~ms}$. Values of at least 20 pixels were chosen for $\mathrm{T} 1$ measurement in each subject. b, $\mathbf{d}, \mathbf{f}$ The color-coded T1 map for postcontrast bladder wall depicts a color gradient indicating a greater shortening of $\mathrm{T} 1$ from GBCA diffusion in pixels representing the inner layer (urothelium) (dark blue color) > pixels representing the middle-layer lamina propria (blue to green) > pixel representing the outer detrusor layer (green to yellow). Larger blue- to green-colored band of pixels in the bladder wall of Hunner-type IC patients indicates deeper bladder wall diffusion of GBCA compared to controls and non-Hunner-type IC/BPS patients. Catheter in the center is shown in (c), and the hip implant of Hunner-type IC patient is indicated 


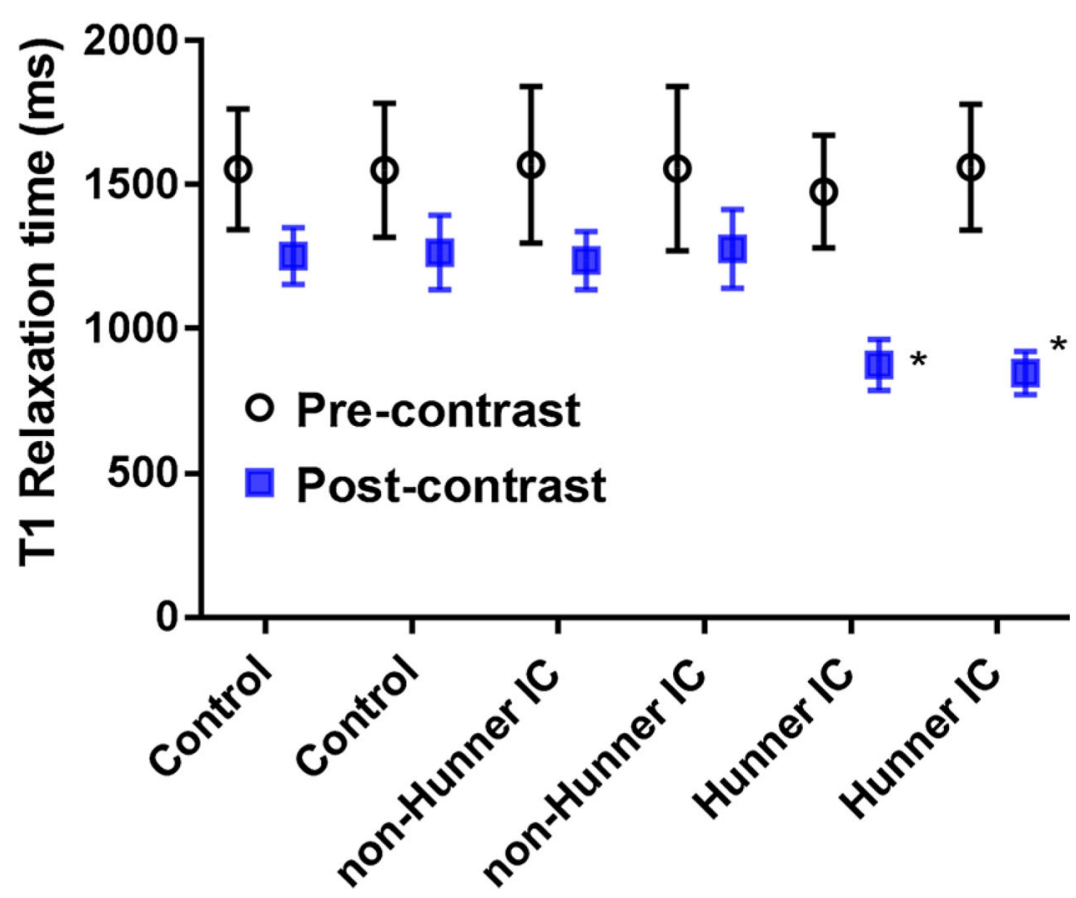

Fig. 6.

The plot shows the pre-contrast and post-contrast values of the bladder wall $\mathrm{T} 1$ in milliseconds (ms). Pre-contrast values of six subjects were not different from each other with a mean value of $1544 \pm 34.2 \mathrm{~ms}$, which was shortened by the diffusion of GBCA into the ECV of bladder wall. Fourfold greater T1-shortening was noted in Hunner-type IC patients with value of $860.09 \pm 13.95 \mathrm{~ms}$ compared to controls and non-Hunner-type IC subjects with values of $1258.16 \pm 6.16$ and $1257.42 \pm 20.59 \mathrm{~ms}$, respectively. Reduction from pre-contrast T1 values (open circles) was 44\% for Hunner-type IC patients compared to only $\sim 18 \%$ for controls and non-Hunner-type IC subjects. Pre-contrast (open circles) and post-contrast (filled square) mean values of individual subjects are shown for comparison and only the statistical difference between post-contrast bladder wall T1 values for individual subjects was assessed (* $p<0.0001$, two-way ANOVA followed by Tukey's test) 